\title{
A 60-year-old male presented with left-sided weakness and difficulty in speech
}

\author{
Iffat Islam Khan, Md. Mahbubul Islam, Redoy Ranjan, Mohammad Tariqul Islam and Asit Baran \\ Adhikary
}

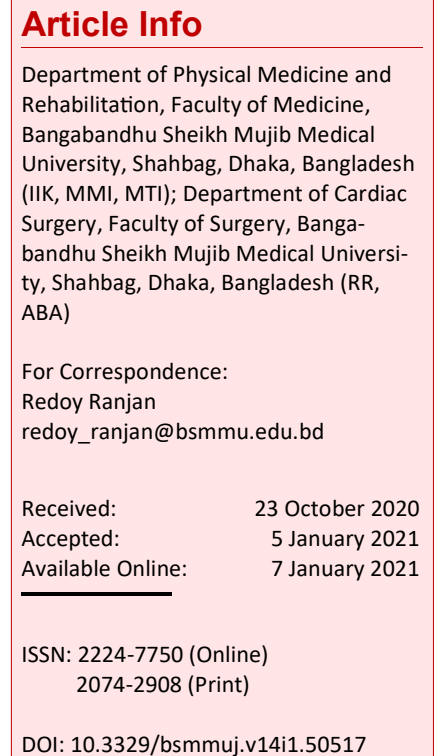

DOI: 10.3329/bsmmuj.v14i1.50517

Cite this article:

Khan II, Islam MM, Ranjan R, Islam MT, Adhikary AB. A 60-year-old male presented with left-sided weakness and difficulty in speech. Bangabandhu Sheikh Mujib Med Univ J. 2021; 14: 712.

\section{Copyright:}

The copyright of this article is retained by the author(s) [Atribution CC-By 4.0]

\section{Available at:}

www.banglajol.info

A Journal of Bangabandhu Sheikh Mujib Medical University, Dhaka, Bangladesh

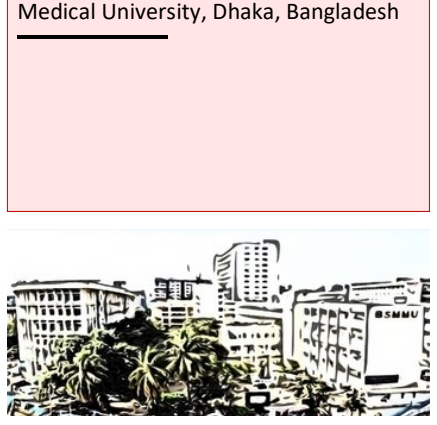

\section{Presentation of Case}

Dr. Iffat Islam Khan (MD Resident): A 60-year-old male presented with weakness of the left side of the body followed by a sudden fall on the ground while working in the field, with difficulty in speech for 03 months. Weakness was sudden in onset which involved left upper and lower limbs with the inability to move the left side of the body and difficulty in walking. The patient also noticed that there was a deviation of the face towards the right side associated with dribbling of food with difficulty in speech but can follow the command with difficulty. There was also urinary incontinence for the same duration, but the bowel movement was normal. There was no history of trauma to the head or spine, convulsion, headache, unconsciousness, vomiting, fever, and double vision. He has been suffering from multivessel coronary artery disease with significant bilateral carotid artery stenosis since 2018. The patient was advised for surgical revascularization with concurrent coronary artery bypass graft and carotid endarterectomy surgery, but he refused. Then he was managed conservatively, but he took his medication irregularly. The patient is hypertensive and has been treated with antihypertensive for the last 5 years. He mentioned having alcohol occasionally. He had no other chronic disease. General examinations revealed normal findings except his pale complexion with moderate anemia. Moreover, cardiovascular system examinations revealed pulse was 75 beats/min (regular), blood pressure $160 / 100 \mathrm{mmHg}$. A carotid bruit present over the both carotid area with the bell of the stethos -cope in a breath-hold position. Examinations of the nervous system revealed normal higher cerebral function; speech was sluggish, upper motor type left-sided facial nerve palsy. On the left upper and lower limb motor system examination, muscle bulk and power (medical research council grade- 2/5) were reduced, muscle tone was increased, and deep tendon reflexes were exaggerated as well as planter reflex was extensor. On musculoskeletal system examination, the patient was unable to walk, mild flexion deformity in the left elbow and wrist with restrictions on the movement par- tially flexed left foot, and external rotation of the left hip joint.

The patient was further investigated with hematological, biochemical, microbiological as well as radiological investigations (Table I). Hematological investigations demonstrated hemoglobin level (\%) was $10.8 \mathrm{~g} / \mathrm{dL}$ and erythrocyte sedimentation rate- $55 \mathrm{~mm}$ in 1st hour. Biochemical investigations showed serum cholesterol- $469 \mathrm{mg} / \mathrm{dL}$, low density lipoprotein$271 \mathrm{mg} / \mathrm{dL}$, as well as triglycerides- $280 \mathrm{mg} /$ dL. Serum electrolytes, Serum creatinine, and random blood sugar were within normal limits. A computed tomography scan scan of the brain showed a hypodense area in the right thalamoganglionic region of the brain (Figure 1). Moreover, electrocardiogram had features of old myocardial infarction (MI) in II, III, aVF leads, and echocardiography showed left ventricular dysfunction (ejection fraction- 40\%) with regional wall motion abnormality. Duplex ultrasonogram of neck vessels showed $60 \%$ and $80 \%$ stenosis of the left and right internal carotid artery, respectively. Coronary angiography demonstrated triple vessel disease where $80 \%$ stenosis in the left anterior descending artery, $70 \%$ stenosis in the left circumflex artery, and $90 \%$ stenosis in the right coronary artery.

Based on history, proper physical examination, and adequate investigations, we would like to draw a provisional diagnosis.

\section{Provisional Diagnosis}

Ischemic stroke due to bilateral carotid artery stenosis with triple vessel disease

\section{Differential Diagnosis}

Intracranial space occupying lesion (ICSOL)

\section{Chronic subdural hematoma}

Dr. Mohammad Tariqul Islam: The term 'ICSOL' includes lesion which expands in volume to displace normal neural structures and lead to an increase in intracranial tension. This lesion causes irritation or destruction of brain tissue, 


\begin{tabular}{|c|c|c|}
\hline \multicolumn{3}{|c|}{ Table I } \\
\hline \multicolumn{3}{|c|}{ Laboratory investigations } \\
\hline Investigation & Findings & Reference \\
\hline Hemoglobin (g/dL) & 10.8 & $15 \pm 2$ \\
\hline $\begin{array}{l}\text { Erythrocyte sedimentation rate (mm } \\
\text { in 1st hour) }\end{array}$ & 55 & $0-10$ \\
\hline \multicolumn{3}{|l|}{ Complete blood count } \\
\hline Red blood cell (x1012/L) & 3.70 & $4.5-5.4$ \\
\hline WBC count $\left(\times 10^{9} / \mathrm{L}\right)$ & 10.5 & $7 \pm 3$ \\
\hline Neutrophil (\%) & 72 & $50-70$ \\
\hline Lymphocyte (\%) & 22 & $20-40$ \\
\hline Monocyte (\%) & 5 & 2-8 \\
\hline Eosinophil (\%) & 1 & $1-4$ \\
\hline Basophil (\%) & 0 & $0-4$ \\
\hline Platelet count $\left(\times 10^{9} / \mathrm{L}\right)$ & 350 & $150-500$ \\
\hline Serum creatinine $(\mathrm{mg} / \mathrm{dL})$ & 0.6 & $0.3-1.0$ \\
\hline Fasting blood sugar (mg/dL) & 5.3 & $<5.5$ \\
\hline 2 Hour after breakfast (mmol/L) & 6.5 & $<7.8$ \\
\hline $\operatorname{HbA1C}(\%)$ & 6.2 & $4.0-5.9$ \\
\hline \multicolumn{3}{|l|}{ Serum lipid profile } \\
\hline Total cholesterol (mg/dL) & 296 & $150-200$ \\
\hline Low-density lipoprotein $(\mathrm{mg} / \mathrm{dL})$ & 271 & $50-130$ \\
\hline Triglycerides $(\mathrm{mg} / \mathrm{dL})$ & 181 & $60-150$ \\
\hline \multicolumn{3}{|l|}{ Serum electrolytes } \\
\hline Sodium (mmol/L) & 134 & $135-145$ \\
\hline Potassium (mmol/L) & 4.3 & $3.5-5.0$ \\
\hline Chloride (mmol/L) & 100 & $96-106$ \\
\hline $\mathrm{T}-\mathrm{CO}_{2}(\mathrm{mmol} / \mathrm{L})$ & 23 & 23-29 \\
\hline
\end{tabular}

such as focal seizures and paralysis, the feature of increase intracranial pressure and false localizing signs.1, 2 A space-occupying lesion characterized by Persistent headache felt in the midline not due to any other detectable cause and unresponsive to simple medication. It may continuous and progressive, paroxysmal, aggravated by coughing, stooping forward, or changing posture. Associated symptoms of space-occupying lesion are vomiting, visual loss, motor and sensory defect, and personality disorder. In most of the cases presented with papilloedema, however, it depends upon the location and mass effect of ICSOL. $\underline{2,3}$ Patients may also present with bradycardia, hypertension, irregular respirations, ipsilateral pupillary enlargement, and loss of light reflex due to compression of the 3rd cranial nerve. Computed tomography scan is capable of disclosing not only a tumor mass, its location, and extension but also any associated pathological changes such as brain edema around the mass, hydrocephalus, hemorrhage, cystic formation, calcification, etc. Magnetic resonance imaging allows a more detailed visualization than computed tomography scan. $\underline{2-4}$

Dr. Redoy Ranjan: A chronic subdural hematoma in an old clot of blood on the surface of the brain beneath its outer covering most often occurs in a patient with the age of 60 years and older having brain atrophy. 5 When the brain becomes atrophied inside the skull, even minor injury can cause tearing of blood vessels over the cerebrum which resulting in a slow accumulation of blood over several days to weeks. Other risk factors include alcoholism, seizures, excess cerebrospinal fluid drainage by shunts from the brain, and blood-thinning medications. $\frac{4,5}{5}$ The most common complaint is a headache, accompanied by lethargy, vomiting, memory loss, fluctuating level of confusion, impaired vision, and seizures. A chronic subdural hematoma may mimic many other brain diseases, including dementia, stroke, and temporary disruption of blood supply to a portion of the brain (TIA), encephalitis, and brain lesions such as trauma or abscess. Computed tomography scan shows hematomas are usually hypodense concavo-convex lesions. But isodense or mixed density lesions are also observed. Magnetic resonance imaging could help in making a diagnosis that is more sensitive than a computed tomography scan in determining the size and internal structures. Fresh bleeding, hemolysis, and hemoglobin changes can also be observed by magnetic resonance imaging. $\underline{1,4-6}$

\section{Dr. Khan's Diagnosis}

Ischemic stroke with left-sided hemiplegia

\section{Discussion}

Dr. Islam: Stroke occurs when a blood vessel in the brain ruptures and bleeds or when there is a blockage in the blood supply to the brain. The rupture or blockage prevents blood and oxygen from reaching the brain tissue. $\frac{5,6}{6}$ Without oxygen, brain cells and tissue became damaged and begun to die within minutes. Stroke symptoms include paralysis, numbness or weakness in the face, arm, leg, especially one side of the body, slurring of speech, vision problem, and difficulty in walking, loss of balance and coordination, severe sudden headache. Stroke requires immediate medical attention; prompt patient management is the key to preventing brain damage, long term disabilities, and death. $\frac{3-5}{5}$ Stroke is mainly categorized into 2 types; Ischemic stroke and hemorrhagic stroke. The two most common types of ischemic strokes arethrombotic and embolic. During an ischemic stroke, the artery supply blood to the brain become narrow and blocked. This blockage is caused by blood clots or reduction of blood flow due to thrombotic or embolic manifestations. The cause of stroke depends on the type of stroke. A Transient ischemic attack is caused by a temporary spasm in an artery to the brain and after a few minutes to a few hours, 


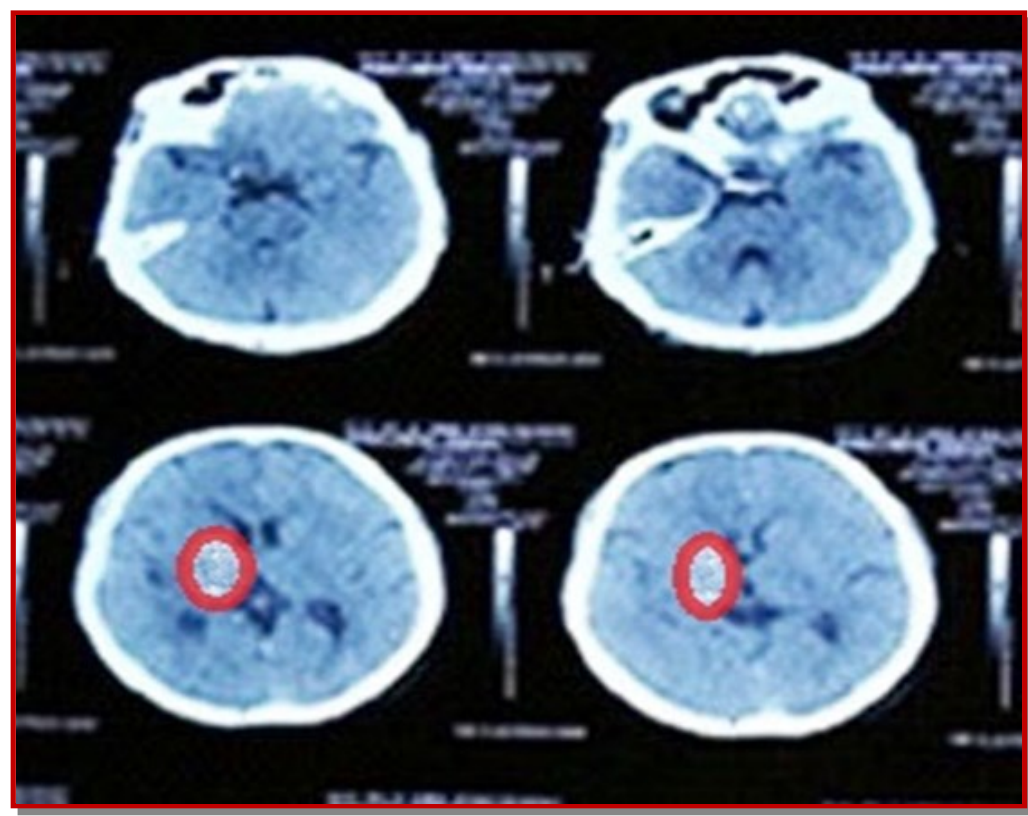

Figure 1: Computer tomography scan (axial view) of brain illustrates a large hypodense area in the right thalamoganglionic region (red circle) which demonstrates plausibility of ischemic stroke

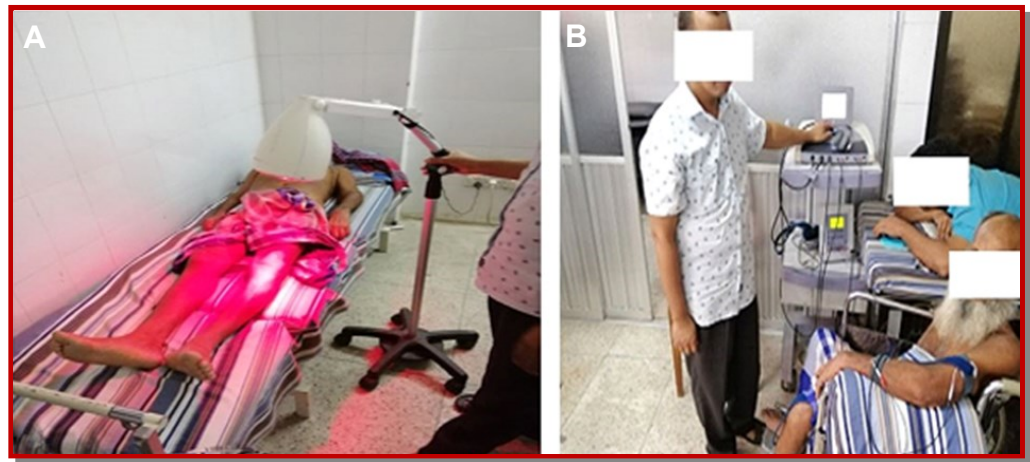

Figure 2: Patient receiving infra-red radiation therapy (A) and electrical stimulation therapy of disability from stroke is about 485 per 10,000 people. 6 Carotid artery stenosis is one of the strong leading causes of ischemic stroke. Carotid arteries supply the frontal $2 / 3 \mathrm{rd}$ of the brain. Atherosclerosis commonly affects carotid arteries and has shown a higher tendency to form in the bulb area. Strokes occur due to blockage at the level of the bulb of the internal carotid artery..$\underline{3-5,9}$

Risk factors for carotid atherosclerosis are old age, obesity, smoking, hypertension, diabetes mellitus, sedentary lifestyle, and hypercholesterolemia. Carotid stenosis remains asymptomatic before it has caused a stroke. Less than $75 \%$ of asymptomatic carotid stenosis carries a $1.3 \%$ chance of stroke per year and $70 \%$ or greater symptomatic carotid stenosis carries an annual stroke risk of $10-15 \%$. There are 3 stroke preventive options in carotid stenosis, which are carotid endarterectomy, carotid stenting, and conservative management with lifestyle modifications. In the management of carotid stenosis, a major concern is the possibility of having complications like perioperative stroke or death which is about $2.3 \%$ and $4.1 \%$ in CEA and carotid stenting, accordingly. $\underline{10-12}$ So, carotid endarterectomy is much safe and feasible. However, when an invasive procedure is contraindicated or undesirable for any reason, aggressive medical management with a statin and anti-platelet drug is the next option. $\frac{5,8-11}{}$

Dr. Md. Mahbubul Islam: How will you evaluate a case of left-sided hemiplegia with speech difficulty to reach the diagnosis?

Dr. Khan: For diagnosis, we need to achieve proper medical history, do a physical examination as well as hematological, biochemical, microbiological, and radiological investigations. History of past medical records like ischemic heart disease, trauma, head injury, blurring of vision, personal history, history of hypertension, diabetes mellitus (insulin resistance), obesity, headache, family history of Atherosclerosis either coronary artery disease or carotid artery disease should be taken. $.4,6,10$ Examination of the nervous system and fundoscopy. Examination of the musculoskeletal system and cardiovascular system including carotid bruit. Routine hematological, biochemical, microbiological as well as radiological investigations, duplex ultra-sonogram of neck vessels. Here, patient has sudden onset of left-sided hemiplegia, difficulty in speech, upper motor neuron type of left-sided facial nerve palsy, there is increased muscle tone in the form of spasticity with exaggerated reflux in left side furthermore computed tomography of the brain showed hypodensity area present in the right thalamoganglionic region all are indicate a case of ischemic stroke with left-sided hemiplegia. $\frac{1,3-7}{-3}$

Dr. Ranjan: What are the management plans for carotid artery stenosis in this case? 
Prof. Adhikary: Carotid artery stenosis accounts for at least $50 \%$ of ischemic stroke and should be managed efficiently to minimize the incidence of stroke. The high-risk patient presented within a few hours of the onset of symptoms of TIA or stroke should undergo urgent duplex scanning. If a $>70 \%$ stenosis is detected in a carotid artery, the patient should immediately be evaluated by vascular surgeons for carotid interventions within 48 hours of presentation. Patients who present after 48 hours, but within 7 days should have carotid endarterectomy within 7 days of onset of symptoms. $\underline{10}$

The patient presenting between 7-30 days after transient ischemic attack/stroke should have surgery within 30 days. The patient presenting between 3090 days after transient ischemic attack (TIA)/stroke should have surgery within 90 days. Recent studies have shown that high-risk patients are likely to benefit from carotid endarterectomy as early as possible up to 90 days after an initial TIA/stroke. Best medical management with a combination of antiplatelet and anticoagulants. With clopidogrel, aspirin, and warfarin therapy have been shown to reduce adverse effects of atherosclerotic stenosis and prevent the progression of symptoms. Moreover, the risk of stroke reduces by $25 \%$ and $32 \%$ by using lipid-lowering agents and angiotensin converting enzyme inhibitor medication, accordingly. Management of hypertension and diabetes mellitus, smoking cessation also significantly reduces the risk of stroke by $48 \%$. Carotid angioplasty and stenting are also indicated for high-risk patients for endarterectomy. This patient has already suffered from triple vessel disease with bilateral carotid artery stenosis for one year. So, combined coronary artery bypass grafting and carotid endarterectomy can be done in a single setting. $\underline{9-13}$

Dr. Khan: Is it possible to be done coronary artery bypass grafting and carotid endarterectomy in a single sitting?

Dr. Ranjan: Concurrent carotid endarterectomy and CABG is feasible and safe with a skilled surgical team. At first carotid endarterectomy is done under general anesthesia. First chest open by standard median sternotomy, then pericardiotomy was done and heart is exposed and stabilized by a retractor. Then neck incision given just anterior to the sternocleidomastoid muscle, then exposing the common, internal, and external carotid artery.10-14 Incision and proper exposure of the carotid bifurcation area is made followed by systemic heparinization. Then carotid artery is clamped and arteriotomy is done by a longitudinal incision. Then atherectomy is done from the carotid artery. The arteriotomy was closed by continuous sutures. Following CEA and off-pump CABG is done by either arterial or venous conduit. After operation, protamine will be given to reverse the action of systemic heparinization. $\underline{12,15,16}$
Dr. Islam: How can a physiatrist help this patient for alignment of his symptom?

Dr. Khan: Rehabilitation of stroke patient is a continuum starting within day's onset and ending only when it no longer produces any positive effect.15-17 When a patient has a persisting major continuing impairment such as hemiplegic with disabilities the rehabilitation component of care is the main focus of management. The comprehensive rehabilitation management program is characterized by a holistic approach with a multidisciplinary team member. Firstly, reassurance and counseling are the keys to management. 16,17 Pharmacological therapy, including symptomatic management, control of hypertension, and dyslipidemia should be done. Use of aspirin or clopidogrel as an antiplatelet agent, cerebral vasodilator, and others. In rehabilitation, management includes proper bed positioning, a therapeutic exercise in the form of passive range of motion exercise, left upper and lower limb stretching exercise, Achilles tendon stretching exercise, proprioceptive neuromuscular facilitation exercise of left facial muscle. all exercise should be done 10 repetitions 3 times daily. Therapeutic modalities are Infrared radiation for 20 minutes daily over the affected part of the body and Electrical stimulation therapy for 15 minutes per day (Figure 2). Other therapies are speech therapy, cognitive therapy, occupation therapy, swallowing therapy, and bladder rehabilitation management program. Early diagnosis with immediate referral and multidisciplinary team management may prevent further progression of disabilities, complications, and minimize the impairments. $\underline{18-20}$

Dr. Ranjan: What is the role of electrical stimulation therapy to improve the muscle power of this patient?

Dr. Islam: Electrical stimulation stimulates the motor unit and causes muscle contractions. This helps in regaining the properties of muscle and boost Neuroplasticity after stroke by increasing the stimulation and send it to the brain. Electrical current is applied through electrodes to one or more targeted muscles.12-15, 18 The muscle size, the position of the electrodes, and the amount of intervening tissue between muscle and skin dictate the intensity needed from the electrical stimulation unit to cause a contraction. Electrical stimulation therapy was given to this patient in the form of a faradic current. A frequency usually ranges between $10-100 \mathrm{Hzs}$. Pulse cycle duration 150-200 microsecond for small muscle and 200-350 microsecond for large muscle, waveform asymmetric and given muscles are left deltoid, triceps, supraspinatus, infraspinatus, wrist extension, ankle dorsiflexion daily for ten minutes. This should continue 10-15 min once or twice per day, 3-6 times per week for 6-12 weeks.

Dr. Islam: What is the role of carotid endarterectomy 
for this patient?

Prof. Adhikary: Carotid endarterectomy is thought to reserve cognitive impairment in patients with highgrade internal carotid artery stenosis by reversing cerebral perfusion. Carotid endarterectomy is effective for symptomatic patients with $70-99 \%$ stenosis and age between $40-70$ years. Which was absolutely indicated for this patient. Accepted practice in many centers is to wait 4-6 weeks after the onset of deficit before proceeding with carotid endarterectomy. It is due to the fear that early revascularization will increase the size of the infracts. $10,23-24$

Dr. Khan: What are the complications we should keep in mind in carotid endarterectomy?

Dr. Ranjan: Some possible complications during carotid endarterectomy includes:12, 22-26 Stroke or transient ischemic attack secondary to dislodgement of a plaque, myocardial infraction, cranial nerves injury especially vagus, hypoglossal and marginal mandibular nerve, intracerebral hemorrhage, restenosis of the carotid artery, infection and compression of airway from swelling or bleeding into the neck.

Dr. Ranjan: How infrared radiation works in the reduction of spasticity and pain?

Dr. Islam: Infra means below or beyond. In the electromagnetic spectrum, the radiation band which falls just below the visible red is called infra radiation. Therapeutic use of this Infrared radiation as superficial dry heat modalities in various clinical conditions is known as infrared radiation therapy. It is radiation heat. The wavelength is long varying from $7700 \mathrm{~A}-125000 \mathrm{~A}$ and low frequency. Time is required about $15-30 \mathrm{~min}$ (15 for subacute and 30 for a chronic condition)..$\underline{.11-25,27}$

The infrared radiation treatment produces a heating effect in the superficial epidermis and dermis that causes vasodilatation which increases blood circulation. This provides oxygen supply and nutrition supply in that area leading to drain waste products that relieve pain. Mild heating by infrared radiation causes relaxation of the muscle and thus relieving muscle spasm. Relief of pain also includes relaxation in the muscles and helps to relieve muscle spasms associated with injury and inflammation. $\underline{23-}$ $\underline{28}$

Prof. Adhikary: Treatment of ischemic stroke needs multi-disciplinary team involvement, which is often missed by the patients and sometimes from doctors also. That can linger treatment cost, a disability could get worsen. Timely referral with proper counseling is needed. To summarize, this was a case of ischemic stroke due to carotid stenosis with multivessel coronary artery disease. Although this patient was initially referred for concurrent carotid endarterectomy and coronary artery bypass graft, the attendant and patient refused to do it, and later admitted with progressive atherosclerosis involving multiple vascular system and associated complications. A simple deviation of the path can cause major damage as we can see in this case. Ideal options for this case are to treat with either concurrent or staged carotid intervention and coronary artery bypass graft surgery which is concordance to other published articles.10, 22, 25-28

\section{Final Diagnosis}

Ischemic stroke with left-sided hemiplegia

\section{Conflict of Interest}

Authors declare no conflict of interest

\section{References}

1. Chalela JA, Kidwell CS, Nentwich LM, Luby M, Butman JA, Demchuk AM, Hill MD, Patronas N, Latour L, Warach S. Magnetic resonance imaging and computed tomography in emergency assessment of patients with suspected acute stroke: A prospective comparison. Lancet 2007; 369: 293-308.

2. Boon PA, Williamson PD, Fried I, Spencer DD, Novelly RA, Spencer SS, Mattson RH. Intracranial, intraaxial, space-occupying lesions in patients with intractable partial seizures: An anatomoclinical, neuropsychological, and surgical correlation. Epilepsia. 1991; 32: 467-76.

3. Datta PK, Sutradhar SR, Khan NA, Hossain MZ, Sumon SM, Hasan I, Datta R, Anwar AT, Islam R, Elahi ME. Clinical Pattern of Intracranial Space Occupying Lesion In Tertiary Hospital. J Dhaka Med Coll. 2019; 28: 17-22.

4. Ko BS, Lee JK, Seo BR, Moon SJ, Kim JH, Kim SH. Clinical analysis of risk factors related to recurrent chronic subdural hematoma. J Korean Neurosurg Soc. $2008 ; 43$ : 11-15.

5. Cenic A, Bhandari M, Reddy K. Management of chronic subdural hematoma: A national survey and literature review. Can J Neurol Sci. 2005; 32: 501-06.

6. Islam MN, Moniruzzaman M, Khalil MI, Basri R, Alam MK, Loo KW, Gan SH. Burden of stroke in Bangladesh. International journal of stroke. 2013; 8: 211-13.

7. Siddique MA, Nur Z, Mahbub MS, Alam MB, Miah MT. Clinical presentation and epidemiology of stroke: A study of 100 cases. J Med. 2009; 10: 86-89.

8. Duncan PW, Zorowitz R, Bates B, Choi JY, Glasberg JJ, Graham GD, Katz RC, Lamberty K, Reker D. Management of adult stroke rehabilitation care: A clinical practice guideline. Stroke 2005; 36: 100-43. 
9. Dev K, Joshi M. Functional outcome of stroke patients, correlation with scandinavian stroke scale. Age Journal. 2013; 40: 13-19.

10. Louridas G, Junaid A. Management of carotid artery stenosis. Update for family physicians. Can Fam Physician. 2005; 51: 984-89.

11. Wang Q, Zhou M, Zhou Y, Ji J, Raithel D, Qiao T. Effects of carotid endarterectomy on cerebral reperfusion and cognitive function in patients with high grade carotid stenosis: A perfusion weighted magnetic resonance imaging study. Eur J Vasc Endovasc Surg. 2015; 50: 5-12.

12. Allain R, Marone LK, Meltzer J, Jeyabalan G. Carotid endarterectomy. Int Anesthesiol Clin. 2005; 43: 15-38.

13. Lanzino G, Rabinstein AA, Brown RD Jr. Treatment of carotid artery stenosis: medical therapy, surgery, or stenting?. Mayo Clin Proc. 2009; 84: 362-68.

14. Antunes PE, Anacleto G, Oliveira JMF. Staged carotid and coronary surgery for concomitant carotid and coronary artery disease. Eur J Cardiothorac Surg. 2002; 21: 181-86.

15. Ranjan R, Adhikary D, Saha H, Mandal S, Saha S, Adhikary A. Concurrent carotid endarterectomy and off-pump coronary artery bypass graft surgery in Bangladesh: A prospective cohort study. Faridpur Med Coll J. 2018; 13: 2-7.

16. Ranjan R, Adhikary D, Mandal S, Saha H, Saha S, Adhikary A. Outcome of combined carotid endarterectomy and off-pump coronary artery bypass grafting surgery in Bangladesh. Int J Recent Sci Res. 2017; 8: 18442-46

17. Maulden SA, Gassaway J, Horn SD, Smout RJ, DeJong G. Timing of initiation of rehabilitation after stroke. Arch Phys Med Rehabil. 2005; 86: 3440 .
18. Rahman MM, Suut N, Putit Z, Arif MT, Said AB, Suhaili MRB, Fadzillah M, AkBakon F, Tambi ZB, Kee LH. Burden of stroke caregivers: Evidence from a qualitative study in Sarawak, Malaysia. BJMS. 2018; 17: 593-99.

19. Mahmood K, Hossain MS, Rahman S. Review of rehabilitation of ischaemic stroke patients. J Dhaka Med Coll. 2009; 18: 169-73.

20. Noll SF, Roth EJ. Stroke rehabilitation. 1. Epidemiologic aspects and acute management. Arch Phys Med Rehabil. 1994; 75: 38-41.

21. Hara Y. Rehabilitation with functional electrical stimulation in stroke patients. Int J Phys Med Rehabil. 2013; 1: 1-6.

22. Kawashima N, Popovic MR, Zivanovic V. Effect of intensive functional electrical stimulation therapy on upper-limb motor recovery after stroke: Case study of a patient with chronic stroke. Physiother Can. 2013; 65: 20-28.

23. Holten KB. Endarterectomy for carotid artery stenosis: Who qualifies? J Fam Pract. 2006; 55: 866-67.

24. Chabowski M, Grzebien A, Ziomek A, Dorobisz K, Lesniak M, Janczak D. Quality of life after carotid endarterectomy: A review of the literature. Acta Neurol Belg. 2017; 117: 829-35.

25. Yang L, Liu J, Qi G, Li Y, Liu Y. The middle-term outcome of carotid endarterectomy and stenting for treatment of ischemic stroke in Chinese patients. Sci Rep. 2018; 8: 4697.

26. Aldoori MI, Baird RN. Local neurological complication during carotid endarterectomy. J Cardiovasc Surg (Torino). 1988; 29: 432-36.

27. Tsai SR, Hamblin MR. Biological effects and medical applications of infrared radiation. J Photochem Photobiol B. 2017; 170: 197-207. 